\title{
Design of Intelligent Curtain Control Circuit Based on Single Chip Microcomputer
}

\author{
Qianqian Zhang *, Jichao Zhao, Haokang Wen, Hongbo Hao \\ Tianjin University of Science and Technology, China; \\ E-mail:577972810@qq.com \\ www.tust.edu.cn
}

\begin{abstract}
Since entering the 21 st century, high technology has promoted the development of human beings and artificial intelligence has been popularized gradually. In this paper, the structure and principle of crystal oscillator circuit, reset circuit and photosensitive sensor circuit are introduced with 89C51 single chip microcomputer as the main control unit. The whole circuit system is analyzed in this paper. The circuit module of photosensitive sensor can be used to detect the external light intensity and automatically control the curtain. The user can set the temperature threshold with the remote control.
\end{abstract}

Keywords: The steering gear 89C51; Photosensitive; Embedded remote control; Temperature monitoring

\section{Overall scheme design}

This design starts from people's demand for the system design function, under the circumstance of comprehensive consideration of various factors, designs the overall framework of the automatic control system, and on the basis of the realization of the overall function, considers the scalability of the system as far as possible ${ }^{1}$.

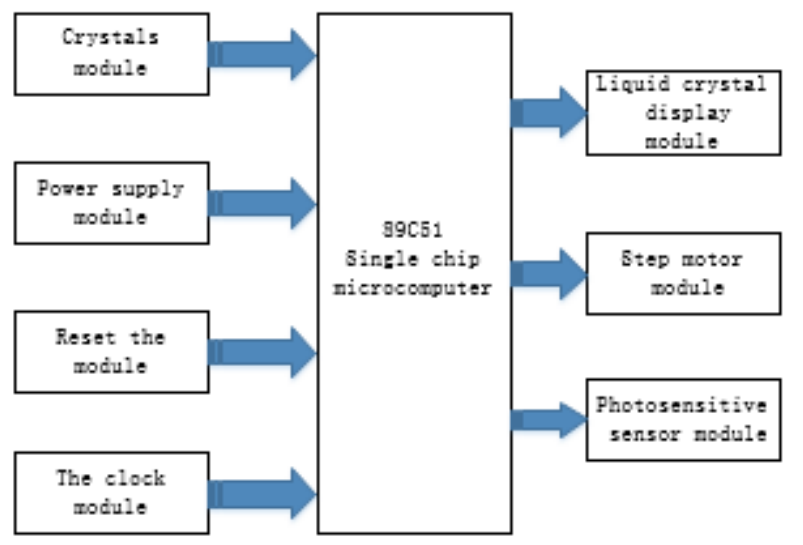

Fig.1. Overall scheme block diagram

\section{Each module scheme design}

(1)Photosensitive control module: This module firstly changes the resistance value of photoresist through the change of the external light intensity, and then causes the change of output voltage. When the light intensity weakens to the set value at night, the light intensity is sensed by the photosensitive sensor, and the stepping motor is controlled by $51 \mathrm{MCU}$ to close the curtain. When the light intensity increases to the set value in the morning, the light intensity is sensed by the photosensitive sensor, and the 51 single-chip microcomputer controls the stepper motor to open the curtain.

(2)Temperature monitoring module: the infrared remote control is used to set the temperature value, and the temperature monitoring system is used to detect the ambient temperature. When the temperature exceeds or is lower than the given value, 51 single-chip microcomputer controls the stepping motor to turn forward to open or close the curtain ${ }^{2}$.

(3)Infrared remote control module: The remote control of this module is realized through the infrared signal sent by THE HT6221 chip. When the receiver receives the infrared signal, the decoding operation is performed first, and then the code value corresponding to each button on the LCD is displayed. The software program allows the user to control the forward and reverse of the motor through the remote control, so that the curtain can be opened or closed. Here, another function of infrared remote control is to set the initial temperature value, and the button can change the initial temperature value, which makes people's life more convenient and comfortable. 


\section{Subsystem design}

\subsection{Circuit design steps}

The crystal oscillator in the circuit is used to generate the reference frequency and optimizes the frequency stability and anti-interference. The accuracy of the frequency in the circuit is controlled by the reference frequency. In addition, it can also produce the clock signal and oscillation current of the microcontroller, the operation of the single chip cannot do without crystal oscillator, it is the peripheral hardware that the single chip system cannot lack, if the circuit is abnormal, the single chip system will be paralyzed, unable to run, leading to the failure of the whole system. All the programs of the single chip microcomputer are written into the read-only memory when they are written.

The single chip microcomputer system must read the program after it is started. The time it takes the processor to read a piece of code is one machine cycle of the processor, and this time is the beat for the processor. Therefore, crystal oscillator circuit is the core peripheral circuit of processor operation. The $12 \mathrm{MHZ}$ vibration source is selected in this design. Although the internal vibration source of the MCU is provided, external vibration source is selected because of the design requirement. Figure2 shows the microcontroller crystal oscillator circuit.

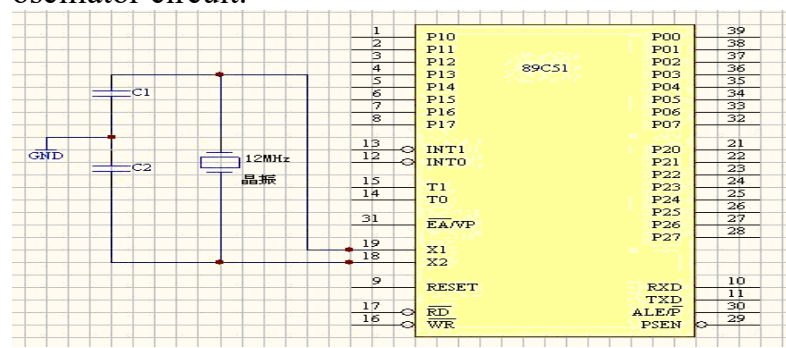

Fig.2.Single chip crystal oscillator circuit diagram

\subsection{Reset circuit}

The processor designed the reset function circuit, which is used to reset the system when the system wants to return to the state of restart. In fact, when the processor starts to run on power, it is a reset state, so the processor startup is required to reset. You will also need to use the reset button to make pin 9 high to restart 89C51 in the "On" or "dead" locked state. Figure3 shows the reset circuit diagram.

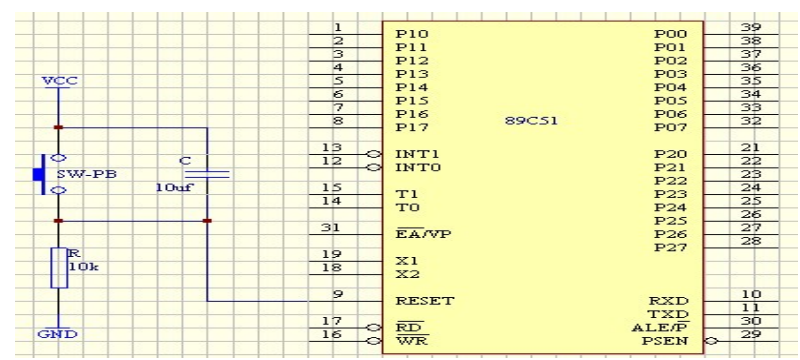

Fig.3.Reset circuit diagram

\section{2 clock circuit}

This design requires the curtain to be opened or closed within a specified time, so a timer is needed. To ensure that the microcontroller is consistent with the external clock, the DS12887 real-time clock chip is used. The chip data can be stored for more than 10 years without damage, and the signal on the RESET pin has no influence on the RAM in the $\mathrm{MCU}^{4}$. The clock circuit diagram is shown in Figure4. RESET is directly connected to the Vcc to ensure that DS12887 enters or exits the power off state. From this pin, it can output a square wave with a frequency of $2 \mathrm{HZ}-256 \mathrm{~Hz}$.

When the interrupt occurs, the microcontroller reads the input signal once or twice to check if the rolloff state has been entered and obtains the three-phase current and voltage at the entire point. MOT grounding, the high 8-bit of DS12887 is set to $7 \mathrm{FH}$, while the low 8-bit is determined by the address of the chip $(00 \mathrm{H}-3 \mathrm{FH})$ in each cell.

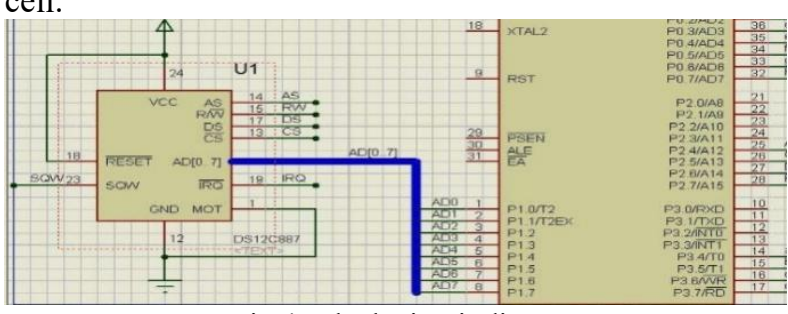

Fig.4. Clock circuit diagram

\subsection{Power circuit}

The operation of the MCU requires $5 \mathrm{~V}$ voltage, so it is necessary to design the power circuit for the MCU. Figure 5 shows the power circuit of the microcontroller.

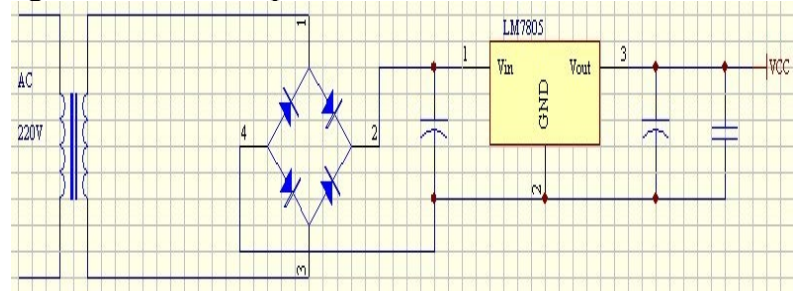

Fig.5. Power circuit diagram 


\subsection{Stepping motor circuit}

Stepper motor is a digital servo actuator with simple structure, reliable operation and convenient control. SCM is controlled by the rotation Angle and displacement of the stepper motor ${ }^{3}$. This design adopts the model of three-phase stepless variable speed motor 130HZ308-450 to control the rotation Angle and displacement. Stepper motor has high torque, high impact resistance and high precision. The drive circuit of the stepping motor operates according to the control signal. Fig.6 and Fig.7 show the block diagram and circuit diagram of the stepper motor control system.

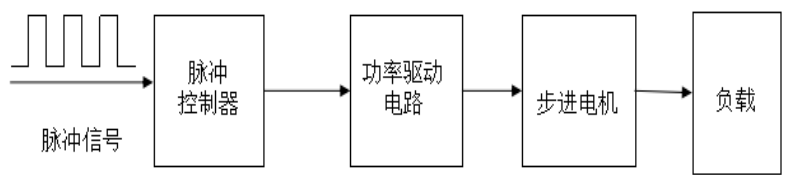

Fig.6. Block diagram of stepper motor control system

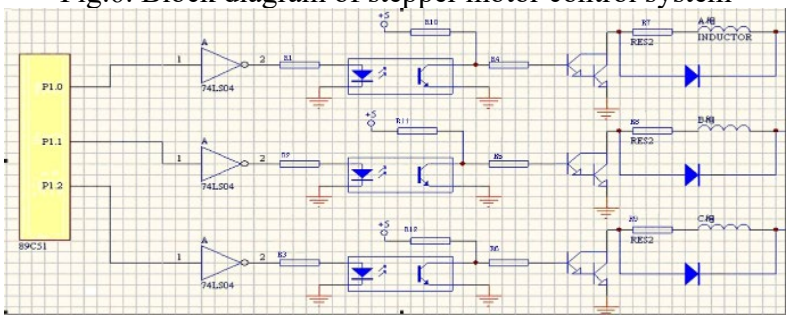

Fig.7.Step motor control system circuit diagram

The pulse distribution circuit is controlled in a predetermined order to open and close the phases. This design not only simplifies the circuit and reduces the cost, but also flexibly changes the control scheme of stepper motor according to the needs of the system. Software control pulse is described in the software design section.

In order to operate in the state of large pulse current, the stepper motor drive circuit works. The photoelectric coupler is used for separation to avoid interference between the SCM and the power supply circuit of stepping motor, and to prevent the main control system from strong electrical interference. In addition, if the driving circuit fails, the MCU at the higher voltage of the power amplifier will not be damaged, and the external resistor of the driving circuit consumes a lot of energy, which will affect the stability of the circuit ${ }^{5}$. Dual voltage drive circuit, as the name implies, has two supply voltages. The current displays on high and low voltage connections, which inevitably results in torque at valley points, which is not easy for motor normal operation to drop.

This disadvantage overcomes the drive to the chopper and the efficiency of the stepper motor can be improved. In this design, constant frequency PWM subdivision drive circuit is adopted, and the block diagram of this circuit is shown in Figure8.

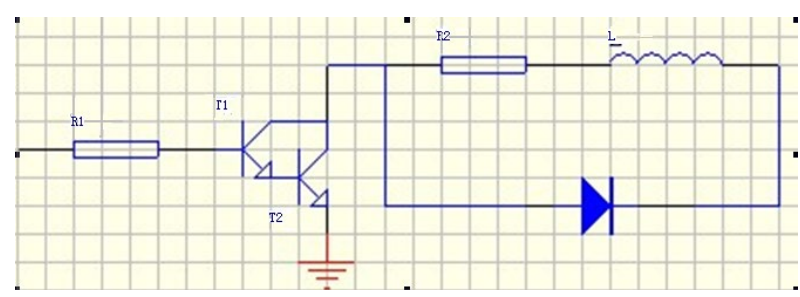

Fig.8.Constant pulse width modulation subdivision drive circuit

In SCM application system keyboard can input data and send instructions to the microcontroller. It is the main means of manual intervention by a microcontroller ${ }^{7}$. When the program is wrong or has an error, you can press the reset key to restore its original working state.

\subsection{LIQUID crystal display circuit}

The connection of liquid crystal module is shown in Figure9. Liquid crystal is used for display in the system. This device can display all characters except Chinese characters, which can fully meet the design requirements. The single chip microcomputer can control the contents of the LIQUID crystal display through the data port and the sequential interface. Through the operation of the software can display any position of the liquid crystal specified display content, easy to operate, simple. The most important is the LCD requirements of the power parameters and SCM system consistent, so that the general power supply, power supply design does not have to reduce design work.

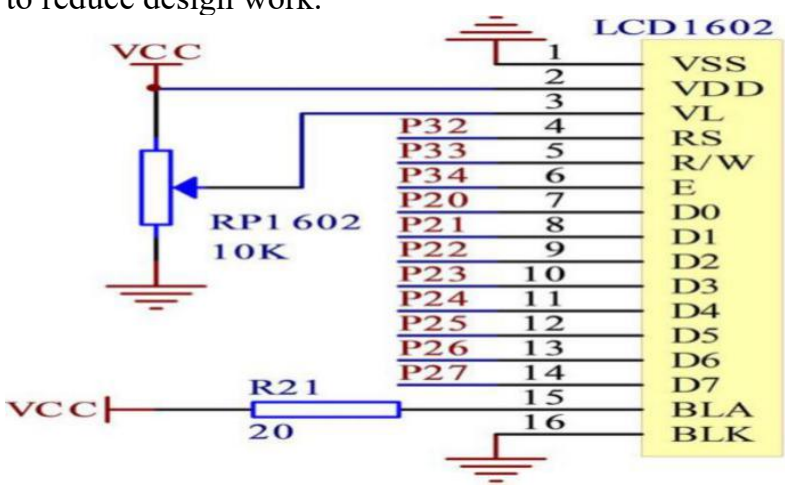

Fig.9.LCD module connection diagram

\subsection{Photosensitive sensor circuit}

The electric curtains are automatically switched on and off depending on the lighting conditions, so photoelectric sensors are required. Photodiode is the main component of photosensitive sensor. It is a photoelectronic device operating on the basis of photoconductor, also known as the light tube ${ }^{8}$. The photodiode has no polarity. It is a purely resistive device. It can be used with a DC offset voltage or ac voltage. When no light is present, the dark resistance of the photodiode is large, and the current in the circuit is small. When there is light irradiation, its photodiode resistance drops sharply, then the current in the circuit increases rapidly.

(C) The 2021 International Conference on Artificial Life and Robotics (ICAROB2021), January 21 to 24, 2021 
Photodiode has the characteristics of high sensitivity and good light splitting. In addition, it is small in size, light in weight and stable in performance. Therefore, it is widely used in automation technology. Photodiodes are commonly used for optical measurement, optical control, and photoelectric conversion (light to electricity). Typically, photodiodes are sliced to absorb more light. When it is illuminated by light, the electron-hole pair is excited to participate in the conduction in the semiconductor wafer (photosensitive layer), reducing the resistance value and thus increasing the current in the circuit.

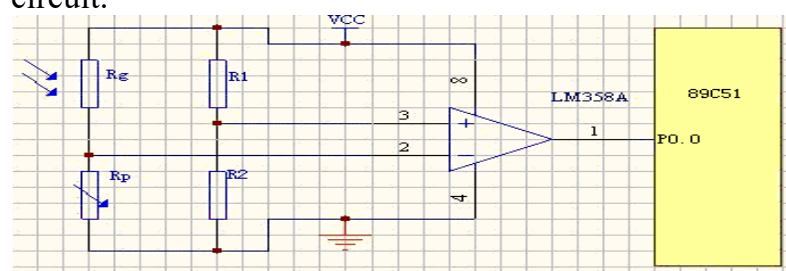

Fig.10.Schematic diagram of optical control circuit

Using the light control principle, the daylight curtain automatically opens and the dark screen automatically closes. The operational amplifier consists of a comparison circuit with two resistors. The two resistors are divided under the same input to obtain a voltage value, which is used to compare with the reference voltage. The reverse input USES a photodiode to collect the light intensity of the external environment. When the indicator light is on, the voltage value of the invert input is constant. Then the voltage values of the two resistors are compared, and the compared signals are sent to the MCU to determine the positive and negative rotation of the motor through the MCU to control the conversion of high and low voltage at port P0, so as to realize the functions of automatic opening of daylight curtains and automatic closing of evening curtains.

In the meantime, we can replace photodiodes with cheaper ones on the market, but the effect is the same. Photodiodes can change their physical properties according to the intensity of light. The signal is changed according to changes in the resistance inside the photodiode by using the unidirectional conductivity of the diode.

\section{Conclusion}

The stepper motor is used as a control component of a single-chip microcomputer to complete the main task of the opening and close the curtain; Photoresistors are used as detection kits to provide external illumination changes for single-chip microcomputers; Infrared detection circuit is used to achieve manual control, the main control chip using 89C51 MCU. In addition, with the help of buttons and display circuits, the intelligent requirements of the automatic curtain control system are finally realized in collaboration with each module.

The sensor part USES photodiodes, which can continuously detect changes in the external light intensity. After the signal from the bridge circuit enters the comparator, a signal can be obtained, and the operation of the stepper motor is controlled by the pulse signal of the single-chip microcomputer ${ }^{7}$.This design of the stepper motor can execute the commands of a single chip very well. Stepper motor is a digital servo actuator, which has the advantages of simple structure, reliable operation, convenient control and good control performance. The designed clock circuit is compatible with the timing function of the single chip microcomputer, and the detection light intensity of the photoelectric sensor solves the problem of automatic control.

\section{References}

1. Li Chaoqing. Principle and Interface Technology of Single Chip Microcomputer. Beijing University of Aeronautics and Astronautics Press, 2013.

2. Li shengli. Fundamentals of analog electronics technology. School of electrical and electronic engineering, wuhan institute of engineering, 2013, 13.

3. Yuan Zengmin. Basic Course of Analog Electronics technology. Beijing: Electronics Teaching and Research Group, Tsinghua University, 2014.

4. Song jingbin. Linux network programming (2nd edition).Beijing: Tsinghua university press, 2014, 2.

5. Liu gang, Zhao jianchuan. Linux system transplantation (second edition). Beijing: Tsinghua university press, 2014, 2.

6. Hu Hancai. Principle of Single Chip Microcomputer and its Interface Technology. Beijing: Tsinghua University Press, 2010: 59-62.

7. Liu Zhuqin, Bai Zesheng. (in Chinese) A Digital frequency meter based on single chip Microcomputer. Modern Electronic Technology, 2010, (1):15-18.

8. Chen Ken, Yang Xiangdong, Liu Li, Yang Dongchao. Robotics and application. Beijing: Tsinghua University Press, 2006. 